\title{
Efficient and Effective Transformed Image Identification
}

\author{
Mohammad Awrangjeb and Guojun Lu \\ Gippsland School of Information Technology, Monash University, Churchill, Vic 3842, Australia \\ \{Mohammad. Awrang jeb, Guojun. Lu\} @ infotech. monash. edu. au
}

\begin{abstract}
The SIFT (scale invariant feature transform) has demonstrated its superior performance in identifying transformed images over many other approaches. However, both of its detection and matching stages are expensive, because a large number of keypoints are detected in the scale-space and each keypoint is described using a 128-dimensional vector. We present two possible solutions for feature-point reduction. First is to down scale the image before the SIFT keypoint detection and second is to use corners (instead of SIFT keypoints) which are visually significant, more robust, and much smaller in number than the SIFT keypoints. Either the curvature descriptor or the highly distinctive SIFT descriptors at corner locations can be used to represent corners. We then describe a new feature-point matching technique, which can be used for matching both the down-scaled SIFT keypoints and corners. Experimental results show that two feature-point reduction solutions combined with the SIFT descriptors and the proposed feature-point matching technique not only improve the computational efficiency and decrease the storage requirement, but also improve the transformed image identification accuracy (robustness).
\end{abstract}

\section{INTRODUCTION}

In many applications, such as image copyright protection [1] and object recognition [2], one common problem is to identify images which may have undergone unknown transformations. We can define this common problem as the transformed image identification (TII), where the goal is to identify the geometric transformed and the signal processed images for a given image. So the TII is different from conventional content-based image retrieval (CBIR) [3], where all images having the same or similar semantic feature, e.g., flower, are considered relevant to each other. The TII consists of three main stages [4]: feature detection, feature representation, and feature matching. In feature detection stage, a set of features, e.g., corners, blobs, T-junctions, are detected. The most valuable property of a feature detector that we consider is repeatability, i.e., whether it reliably finds the same feature points under different viewing conditions of the same image. In feature representation stage, each detected feature point is represented by a feature vector. In feature matching stage, the feature vectors of the test image and the stored images are compared to identify transformed images for the test image. The matching is often based on a distance, e.g., the Euclidean distance [4], between the vectors.

\section{A. Feature Detectors}

Probably the most widely used detector is the Harris interest point detector [5]. However, Harris points are not scale-invariant [4]. Lindeberg [6] introduced the concept of automatic scale selection which allows detecting interest points in an image, each with their own characteristic scale. Mikolajczyk and Schmid [7] refined this technique by creating robust and scale invariant features. Lowe's [2] approximation of the Laplacian of Gaussian using a Difference of Gaussian (DoG) filter speeds up the feature detection stage significantly. The recently proposed Fast-Hessian detector in the SURF (speeded up robust features) detector-descriptor scheme [4] used a basic approximation of the Hessian matrix and relied on the integral images to reduce the computational cost. There are other type of detectors [8], [9] which detect corners on the extracted edges from the gray-scale image.

\section{B. Feature Descriptors}

The SIFT (scale invariant feature transform)-based descriptors, originally proposed by Lowe in [2], have been declared the best among the different types of feature descriptors [10]. The original SIFT descriptor [2] captures a substantial amount of information about the spatial intensity patterns around a feature point. For each descriptor it computes a 3$\mathrm{D}$ histogram of gradient location and orientation, where the location is quantized into a $4 \times 4$ location grid and for each location grid (total 16) the gradient angle is quantized into 8 orientations. The resulting descriptor is a 128-dimensional vector ( 8 orientation bins for each of $4 \times 4$ location bins). This vector is robust to small deformations or localization errors.

Further research has been carried out in the literature either to reduce the descriptor's dimension [4], [11] or to make it more distinctive [10], [11]. Though the lower dimensional PCA-SIFT (principal component analysis-SIFT) [11] and SURF [4] are helpful for fast feature matching, PCASIFT was proved to be less robust than SIFT under affine transformations [10] and SURF was designed to handle rotation and scale attacks only as a compromise between feature complexity and robustness [4]. The GLOH (gradient locationoriented histogram) [10] is another variant of SIFT which was proved to be more distinctive and robust than the original SIFT [10]. However, it is computationally more expensive [4]. Consequently, in spite of the above refinement versions, the original SIFT is still the most popular.

\section{Feature Matching}

Mikolajczyk and Schmid [10] noted three feature matching techniques. In threshold-based matching, two features are matched if the distance between their descriptors is below a 
predefined threshold. A feature may have several matches in this strategy. In nearest-neighbor-based matching, two features $F_{1}$ and $F_{2}$ with descriptors $D_{1}$ and $D_{2}$ respectively are matched if the descriptor $D_{2}$ is the nearest neighbor of $D_{1}$ and their distance is below a threshold. With this approach a feature should have at most one match. In nearest-neighbordistance-ratio-based (NNDR matching) matching, which is similar to nearest-neighbor-based matching, the threshold is applied to the distance ratio between the first and second nearest neighbor matches, i.e., two features $F_{1}$ and $F_{2}$ with descriptors $D_{1}$ and $D_{2}$ respectively are matched if $\left|D_{1}-D_{2}\right| /\left|D_{1}-D_{3}\right|<t$, where $D_{3}$ is the descriptor of the second nearest neighbor match $F_{3}$ of $F_{1}$. In this approach, a feature has also at most one match.

The worst case (as well as the average case and the best case) running time of these algorithms is $O(m n)$, where $m$ and $n$ are the numbers of features in two images. Note that using these matching techniques a repeated feature may be missed or there may be some false positive matches.

\section{This Paper}

In the original SIFT approach [2], a large number of keypoints are detected using DOG filter in the scale-space and each keypoint is represented using a 128-dimensional vector. Therefore, the storage requirement is quite high and all three stages of TII become computationally expensive using the SIFT approach. The reduction in descriptor's dimension [4], [11] resulted in loss of robustness [4], [10]. Instead, the number of the detected feature-points should be reduced to reduce both storage and computational costs.

In this paper, as an aim to reduce the number of featurepoints we propose two solutions. First, we down scale the input image before finding the SIFT keypoints. Second, instead of keypoints we use corner-points, which are visually significant, more robust, and much smaller in number than the SIFT keypoints. Either the curvature descriptor or the highly distinctive SIFT descriptors at corner locations can be used to represent corners. We also propose a new geometric point matching technique. It can be used for matching both the down-scaled SIFT keypoints and corners detected by a contour-based corner detector [8]. Experimental results show that the two featurepoint reduction solutions combined with the SIFT descriptors and the proposed feature-point matching technique not only improve the computational efficiency and decrease the storage requirement, but also improve the TII accuracy (robustness).

\section{Proposed Transformed Image IdentificAtion}

For identifying the transformed images for a given test image, all three stages - feature detection, description, and matching - should be efficient and effective. The original SIFT approach detects a several hundreds to a few thousands of keypoints from a medium size image. Its detection stage is slow because of the use of image scale-space. Moreover, for many applications, e.g., image copyright protection, this huge number of keypoints make the later stages unnecessarily slow.
To overcome this problem, we propose two solutions. First solution is to down scale the input image before DoG keypoint detection. So, the detection stage becomes faster and the number of keypoints is reduced exponentially, which also speeds up the later stages. The highly distinctive SIFT descriptors are used to represent the keypoints and the proposed geometric point matching (GPM) is used for keypoint matching.

As a second solution, we propose using corners instead of SIFT keypoints. In general, corners offer the following advantages over keypoints:

- Corners are visually distinguishable and more robust than their keypoints counterparts.

- In an image, the number of corners is much lower than the number of keypoints.

- Corners can be ranked based on their strength like the curvature value or the number of corners can be controlled by changing the detection thresholds. Therefore, a particular number of strong corners can be selected based on the application and it gives further reduction in computational cost during matching. In contrast, it is very hard to rank the SIFT keypoints.

- Corner detection requires less time than keypoint detection in the scale-space.

Either the curvature descriptor or the highly distinctive SIFT descriptors at corner locations can be used to represent corners and the GPM is used for corner matching.

Consequently, in this paper we propose the following three approaches of TII:

- In the first approach, we use the ARCSS corners as feature-points, the curvature descriptors to represent the corners, and the proposed GPM for corner matching,

- In the second approach, we use the DoG keypoints as feature-points, the SIFT descriptors to represent the keypoints, and the GPM for keypoint matching, and

- In the third approach, we use the ARCSS corners as feature-points, the SIFT descriptors to represent the corners, and the proposed GPM for corner matching.

In the following subsections we will first briefly present the contour-based corner detector [8] (Section II-A). We will then present the proposed point matching technique (Section II-B). Finally, we will present the three aforementioned approaches of TII (Section II-C).

\section{A. Corner Detector}

The affine-resilient curvature scale-space (ARCSS) corner detector [8] extracts edges in the gray-scale image using the Canny edge detector [12]. It then parameterizes each curve (edge) with the affine-length. In order to eliminate noise, it convolves each parameterized curve using the Gaussian kernel in one of three medium scales based on the curve's affinelength. Thereafter, it calculates absolute curvature value on each point of the smoothed curves and considers curvature maxima points as candidate corners. Both weak and false corners are removed using the appropriate thresholds. Finally, corners are tracked down to the finest scale to improve localization. 
The experimental results in [8] showed that in geometric transformations the ARCSS detector outperformed existing CSS detector [9], which outperformed the Harris detector [5]. Note that depending on the applications, the Canny edge detector thresholds set by the ARCSS detector can be changed in order to control the number of detected corners.

\section{B. Geometric Point Matching}

The successful application of a feature detector in many applications depends on how to match feature-points between images. We propose a simple feature-point matching algorithm based on the geometric invariance theory [13].

At least three points must be truly matched to estimate the affine transformation matrix between two images, where one is an affine-transformed version of another. Moreover, the area of a triangle is relatively invariant to affine transformations:

$$
\Delta_{a}=\Delta_{0}\left(s_{x} s_{y}\left(1-s_{h x} s_{h y}\right)\right),
$$

where $\Delta_{0}$ and $\Delta_{a}$ are areas of the original and transformed triangles formed using three points as vertices, $s_{x}$ and $s_{y}$ are scaling factors and $s_{h x}$ and $s_{h y}$ are shearing factors along $x$ and $y$ directions respectively. A function $f$ is called relative invariant to a transformation group $G$ if whenever $f$ is transformed to $F$ by a transformation $g$ in $G$, we obtain $F=z . f$, where $z$ is a function of $g$ alone. If $z \equiv 1$ for all $g$ in $G, f$ is called absolute invariant. For more detail and rigorous discussions see [14].

The proposed geometric point matching (GPM) technique works in following three steps:

- Step 1: We first find the candidate point matches by matching the feature descriptors between two images.

- Step 2: If three candidate matching points are noncollinear on each image and the ratio of areas of corresponding triangles in both the images is within a specific range (depending on the transformation range we want to consider and is calculated using (1); we relax the range a bit since the detected corners may have some localization error), we estimate the transformation matrix between these triangles.

- Step 3: We transform all the corners in one image using the estimated transformation matrix and determine the number of point matches between the two images allowing a localization error of 3-pixels.

Steps 2 and 3 continue over all the combinations of three candidate matches found in Step 1 and track the combination that offers the highest number of point matches.

The worst case running time of this algorithm is $O\left(m^{3} n^{4}\right)$, where $m$ and $n$ are the numbers of feature-points in two images. ${ }^{1}$ However, if the feature descriptor used to represent the feature-point is highly distinctive, the number of false candidate matches between two images will be nearly zero and the algorithm will run much faster. Under this assumption,

\footnotetext{
${ }^{1}$ Finding candidate matches takes $O(m n)$ time. Total number of 3-point combinations is ${ }^{m} C_{3} \times{ }^{n} C_{3}=O\left(m^{3} n^{3}\right)$. For each combination, calculation of transformation matrix and transforming the corners take $O(n)$ time So total time is $O\left(m n+m^{3} n^{4}\right)=O\left(m^{3} n^{4}\right)$.
}

there will be one of the two following scenarios: $(i)$ when two images are related (one is a transformed version of another): there will be maximum $O\left(n^{\prime}\right)$ candidate matches, where $n^{\prime}=\min (m, n)$, and the algorithm runs in $O\left(n^{\prime 4}\right)$ time $^{2}$ ( $\left.i i\right)$ when two images are not related: there should be almost zero number of candidate matches when the algorithm runs in $O(m n)$ time. $^{3}$ In practical TII, the number of first scenario is much lower than the number of second scenario. Hence, the algorithm runs close to $O(m n)$, if the feature descriptor is highly distinctive. So, the challenge with the proposed matching technique is to choose a highly distinctive feature vector to minimize the number of false candidate matches. In this paper, we will use two types of descriptors (curvature and SIFT) and compare their performance.

Though the idea of the proposed matching is similar to that of the RANSAC [15], the former differs the later in the following aspects. First, the proposed matching obtains the candidate matches using the feature descriptors. Since the standard RANSAC does not use such local matching process, it performs poorly when the proportion of the outliers increases above $50 \%$ [2]. Second, the RANSAC sets a termination condition, e.g., terminate the algorithm once a specific number of iterations are executed. We conjecture that if the feature descriptors are distinctive, then the number of false candidate matches will be low and we do not need to set any termination condition. Finally, the RANSAC requires careful setup of a number of algorithmic parameters to ensure its good performance. The proposed matching technique requires only a few parameter setups based on the range of geometric transformations we want to consider.

\section{Proposed TII Approaches}

In this subsection, we will discuss how the proposed GPM technique can be used for matching different feature-points. As discussed before, we can use either the curvature descriptors or the SIFT descriptors to represent the ARCSS corners. We will describe the proposed three approaches of TII below.

1) Matching ARCSS Corners with Curvature Descriptors: In this TII approach, we use ARCSS corners as feature-points, the curvature descriptors to represent corners, and the proposed GPM for matching. Below we will discuss how the curvature descriptor is formed and how the candidate corner matches are obtained using the curvature descriptors.

The ARCSS corner detector makes various information available for later applications. For each corner we get its position, absolute curvature value, angle with its two neighboring corners or with endpoints when enough (two) neighbor corners are not found, and affine-lengths between neighboring corners on the same curve. We will use all the above information collectively as the 'curvature descriptor' to represent the corner. As storing this information per corner takes only a few bytes of memory, the storage requirement for all descriptors per image is very low in this approach.

\footnotetext{
${ }^{2} O(m n)$ to obtain candidate matches and each of $O\left(n^{\prime 3}\right)$ combinations takes $O\left(n^{\prime}\right)$ time to transform corners. So total $O\left(m n+n^{\prime 4}\right)=O\left(n^{\prime 4}\right)$.

${ }^{3} \mathrm{O}(\mathrm{mn})$ to obtain the candidate matches.
} 

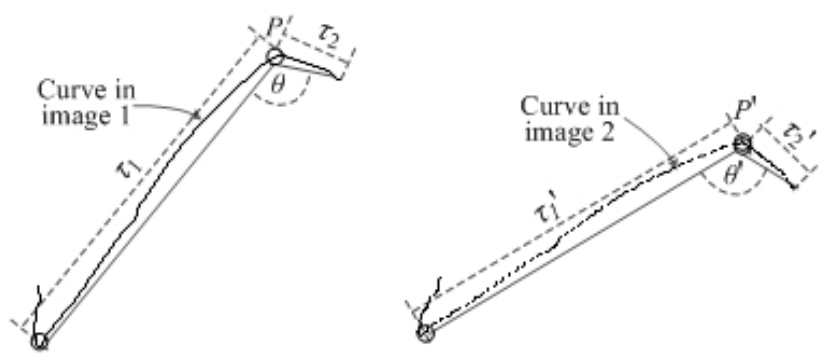

(a) corners on a curve in image

(b) corners on a curve in image 2

Fig. 1. Removing candidate corner matches: let $\left(P, P^{\prime}\right)$ be an initial candidate match consisting of corners $P$ and $P^{\prime}$ between two images. First we check whether two affine-lengths on both sides of these two corners are similar $\left(\tau_{1}\right.$ is compared with $\tau_{1}^{\prime}$ and $\tau_{2}$ is compared with $\tau_{2}^{\prime}$ ). Second we check whether two interior angles $\theta$ and $\theta^{\prime}$ at two corner points are similar. If any of the two above checking fails, the candidate corner match $\left(P, P^{\prime}\right)$ is removed from the candidate corner matching set.

The affine-length between two points on a curve is absolutely invariant to rotations and translations, but relatively invariant to scaling and shearing [8]. Through a comprehensive robustness study ${ }^{4}$ on the ARCSS corners using the benchmark images [16], [17] we observed that the ARCSS curvature values are quite robust under different transformations. The detector gave many repeated corners between original and transformed images within the absolute curvature difference 0.2 (on average 13 and minimum 7 repeated corners) and the absolute angle difference $20^{\circ}$ (on average 15 and minimum 8 repeated corners), when the average number of detected corners was 31 per image.

The candidate corner matches are obtained by comparing the absolute curvature values of corners between two images. Two corners $P$ and $P^{\prime}$ from two images are considered as an initial candidate match if their absolute curvature difference is within 0.2. However, the size of this candidate matching set is quite large and, therefore, we try to remove false candidate matches as explained in Fig. 1. We remove those candidate matches from the set if the corresponding curve affine-lengths on both the sides of the matching corners are not similar (using relative-invariance of affine-length [8]). We also remove those candidate matches whose angle difference is more than $20^{\circ}$.

Once we have the candidate point matching set, we apply steps 2 and 3 of the GPM technique to identify the transformed images.

2) Matching down-scaled DoG Keypoints with SIFT Descriptors: In this TII approach, we use the SIFT descriptors to represent down scaled-DoG keypoints and apply the GPM technique for matching.

In the usual SIFT [2], the DoG detector detects several hundreds to a few thousands of keypoints from a medium size image. Applying the proposed matching technique to such a huge number of keypoints will be very expensive. Moreover, since each descriptor of a keypoint is a 128-dimensional vector

\footnotetext{
${ }^{4}$ Due to page limitation, we only summarize the important information here, instead of detail, of this robustness study.
}

of floating point numbers, storing the descriptor information per image takes a few megabytes of memory, which in turn takes several gigabytes of memory for a moderate database.

To reduce both the matching and storage costs, the images are down scaled before feature detection to reduce the number of keypoints. In this case, only the coarse scale features will be detected which are more robust than the fine scale features.

The candidate keypoint matches are obtained by matching the SIFT descriptors of the detected keypoints using the NNDR matching technique described in Section I-C. Once we have the candidate point matching set, we apply steps 2 and 3 of the GPM technique to identify the transformed images.

3) Matching ARCSS corners with SIFT Descriptors: In this TII approach, we use ARCSS corners, SIFT descriptors at corner locations, and the GPM technique.

In spite of different measures to reduce the number of candidate matches in the first approach (Section II-C1), we observed that the number of false candidate matches is still high which made the matching stage expensive. To overcome this problem, we use the highly distinctive SIFT descriptor, instead of the curvature descriptor, at each corner position while obtaining the candidate corner matching set. This strategy, though, increases the storage requirement, improves the matching performance highly in terms of both efficiency and effectiveness. Similar to the second approach (Section II-C2), the candidate corner matching set is obtained by matching the SIFT descriptors using the NNDR matching technique.

Once we have the candidate point matching set, we apply steps 2 and 3 of the GPM technique to identify the transformed images.

\section{PERFORMANCE STUdy}

We implemented the three proposed approaches on a machine with the following configuration: Dual Core AMD Opteron(tm) Processor $(265 \times 2)$, 4GB RAM, Linux 2.6.18 kernel (Debian Distribution). We used a large database and compared the proposed approaches with the existing most popular SIFT approach [2]. We present the results using the precision-recall graph [3].

\section{A. Database}

We randomly selected 1,050 images from David Nister's recognition database [18]. Each images was of size $480 \times 640$ and converted to gray-scale. Then, for each of the image we applied following 10 simple transformations:

(i) rotation-crop: $\theta=30^{\circ}$,

(ii) scale: $s_{x}=1.2, s_{y}=0.8$,

(iii) rotation-scale: $\theta=20^{\circ}, s_{x}=1.2, s_{y}=0.8$,

(iv) shear: $s_{h x}=s_{h y}=0.012$,

$(v) \quad$ rotation-scale-shear: $\theta=10^{\circ}, s_{x}=1.1, s_{y}=0.9$, $s_{h x}=s_{h y}=0.01$,

(vi) jpeg: quality factor $=20$,

(vii) Gaussian noise: mean $=0$, variance $=0.001$,

(viii) Gaussian blurring: sigma $=3$, window $=3 \times 3$,

(ix) rotation-scale-jpeg: $\theta=20^{\circ}, s_{x}=1.2, s_{y}=0.8$, quality $=20$, 
TABLE I

DIFFERENT TRANSFORMED IMAGE IDENTIFICATION APPROACHES

\begin{tabular}{l|c|c|c}
\hline \hline Approaches & Detectors & Descriptors & Matching \\
\hline Approach 1 & DoG & SIFT & NNDR \\
\hline Approach 2 & scale-DoG & SIFT & NNDR \\
\hline Approach 3 & ARCSS & curvature & GPM \\
\hline Approach 4 & scale-DoG & SIFT & GPM \\
\hline Approach 5 & ARCSS & SIFT & GPM \\
\hline \hline
\end{tabular}

(x) rotation-scale-shear-jpeg: $\quad \theta=20^{\circ}, s_{x}=1.2, s_{y}=$ $0.8, s_{h x}=s_{h y}=0.01$, quality $=20$,

As a result, we had total 1,050 groups of images, each group having 10 relevant images, and in total 10,500 images in the database.

\section{B. Evaluation Metrics}

We used precision and recall [3] collectively to measure the identification performance. Recall measures the system capacity to retrieve the relevant images from the database. It is defined as the ratio between number of retrieved relevant images $r$ and total number of relevant images $T$ (group size) in the database:

$$
\text { Recall }=\frac{r}{T} .
$$

Precision measures the retrieval accuracy. It is defined as the ratio between $r$ and number of retrieved images $R$ :

$$
\text { Precision }=\frac{r}{R} \text {. }
$$

In practice, the performance of an information retrieval system is presented using the precision-recall graph, where the higher the precision at a given recall value the better the performance of the retrieval system [3].

\section{Approaches to be Compared}

In our experiments, we considered five different approaches including the three proposed ones as shown in Table I. Approach 1 is the usual SIFT [2]. In Approach 2, we down scaled the images to $60 \times 80$ in order to reduce the number of detected features in the usual SIFT. In Approach 3, the proposed GPM matching was used with the ARCSS detector where each corner was represented with the curvature descriptor (Section II-C1). In Approach 4, the NNDR matching of Approach 2 was replaced with the GPM technique (Section II-C2). Finally, in Approach 5 the curvature descriptor was replaced with the highly distinctive SIFT descriptor (Section II-C3). Approaches 3 to 5 are our proposed ones and Approach 2 is introduced to compare with the proposed Approach 4.

\section{Experimental Results and Discussions}

Fig. 2 shows the TII performance with different approaches. The graphs are averaged on 16 random queries. Approach 2 performed worse than the usual SIFT (Approach 1) for following two reasons: $(i)$ in Approach 2, though the number of feature-points is decreased to speed up both the detection and matching stages, the number of matching features using the NNDR matching is also decreased; and ( $i i)$ in contrast,

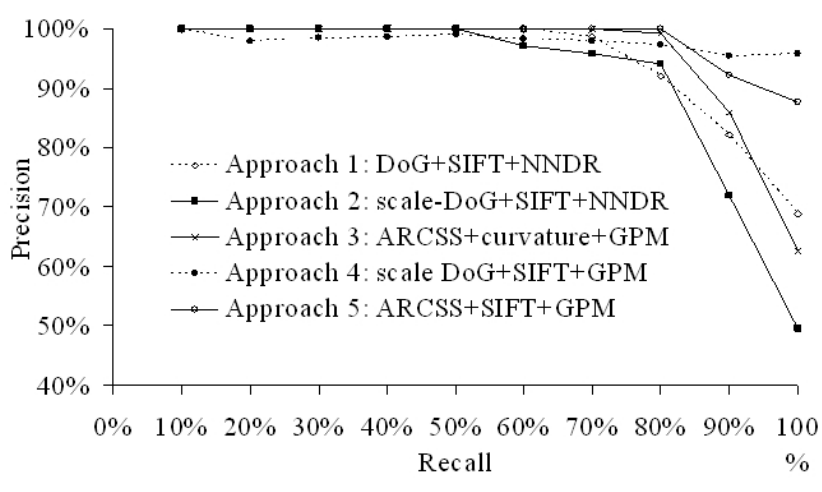

Fig. 2. Transformed image identification performance.

the main reason behind the success of the usual SIFT is, it detects a lot of features and a large number of them are found matched between the relevant images.

Approach 3 required lower storage and total computational costs and showed slightly higher identification accuracy than Approach 1. While the former used the curvature descriptor with the GPM technique, the latter used the SIFT descriptors with the NNDR matching technique. Approaches 4 and 5 outperformed others by representing scale-DoG points and corners respectively with the SIFT descriptors.

Table II summarizes the performance of different approaches. Approach 5, followed by Approach 4, performed the best in terms of both the identification accuracy and total computational cost, though it required higher storage for descriptors than Approach 3. Approach 1 required very high storage for descriptors and its all the stages were quite expensive. The total computational cost and the identification accuracy by Approach 3 was slightly better than the usual SIFT. Moreover, Approach 3 required much lower storage than Approach 1. The storage requirement, total time, and the identification accuracy of Approach 1 were significantly improved by the application of the proposed first feature-point reduction solution (see Approach 4). The total time and the identification accuracy of Approach 3 were also considerably improved by using the distinctive SIFT descriptors at the corner locations, though the storage requirement was increased moderately (see Approach 5, the proposed second featurepoint reduction solution). This proves the high distinctiveness of the SIFT descriptor over the curvature descriptor.

Therefore, by combining the advantages of corners, SIFT descriptors, and GPM technique, Approach 5 can be considered as the overall best.

\section{E. Transformed Image Identification Examples}

Fig. 3 presents a transformed image retrieval example by Approaches 1, 3, and 4. Approach 5 performed the same as Approach 3 (no irrelevant images were retrieved) in this example. In this example, while Approach 1 retrieved 16 and Approach 4 retrieved 2 irrelevant images, Approach 3 retrieved no irrelevant images. The top 10 retrieved images are shown in 
TABLE II

COMPARING PERFORMANCE (PER IMAGE) OF DIFFERENT TRANSFORMED IMAGE IDENTIFICATION APPROACHES IN TABLE I.

\begin{tabular}{c|c|c|c|c|c|c}
\hline \hline Approaches & Features per image & Storage & TII Accuracy (\%) & Detection \& Rep. time (sec) & Matching time (sec) & Total time (sec) \\
\hline Approach 1 & 585 & high & 94.2 & 2.70 & 3.380 \\
\hline Approach 2 & 37 & moderate & 90.9 & 0.54 & \\
\hline Approach 3 & 46 & low & 94.8 & 0.17 & 0.002 \\
\hline Approach 4 & 37 & moderate & 97.9 & 0.54 & 4.330 \\
\hline Approach 5 & 71 & moderate & 98.0 & 0.17 & 0.004 & 0.04 \\
\hline \hline
\end{tabular}

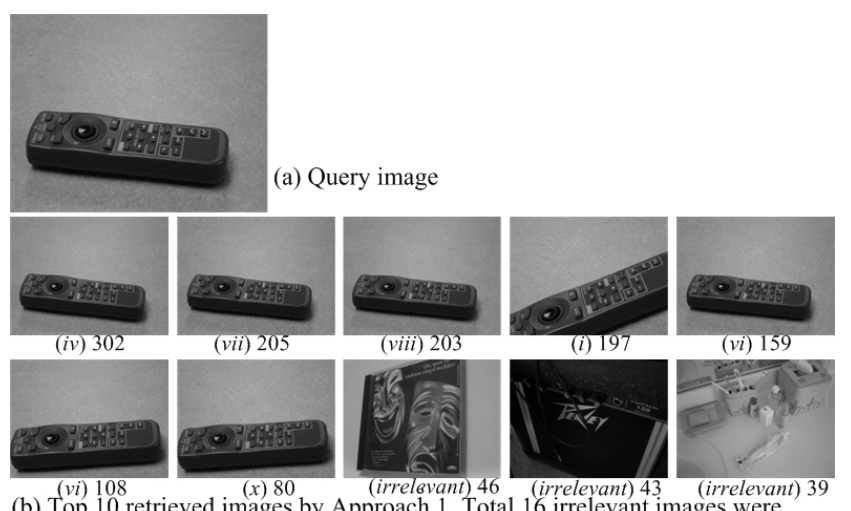

(b) Top 10 retrieved images by Approach 1 . Total 16 irrelevant images were retrieved, 3 of which were in top 10 as shown above.
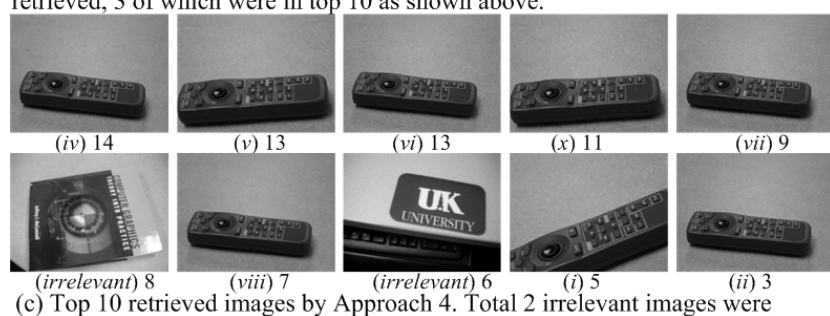

(c) Top 10 retrieved images by Approach 4 . Total 2 irrelevant images were retrieved and they were in top 10 as shown above.

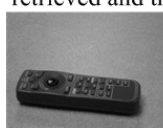

(vii) 25
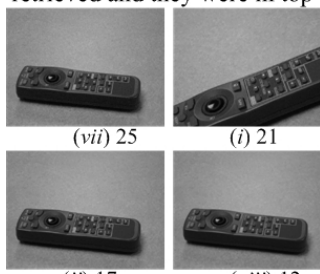

(i) 21

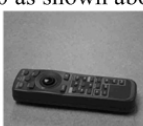

(vi) 20
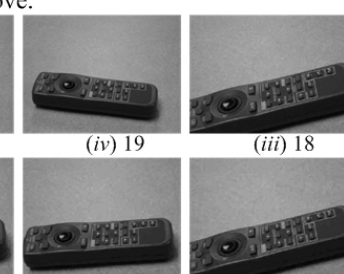
(iii) 18

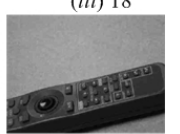

(d) Top 10 retrieved images by Approach 3. No irrelevant image was retrieved.

(d) Top 10 retrieved images by Approach 3 . No irrelevant image was retrieved.

(d) Top 10 retrieved images by Approach 3 . No irrelevant image was retrieved.

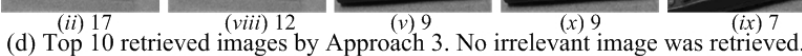

Fig. 3. Transformed image retrieval examples by Approaches 1, 3, and 4 Approach 5 performed the same as Approach 3 (no irrelevant images were retrieved) as shown in (d) above. The number of detected features in the query image by these Approaches were 488, 19, and 37 respectively. The roman number (inside parentheses) under each image indicates a particular transformation discussed in Section III-A and the number after it is the number of feature matches with the query image.

Fig. 3 for each Approach, where 3 irrelevant retrieved images by Approach 1 and 2 by Approach 4 are also shown.

\section{CONCLUSION}

We have shown both the feature detection and matching stages of TII can be significantly speeded up by reducing the number of detected feature-points. We have presented two feature-point reduction solutions. First is to down scale the image before the DoG keypoint detection and second is to use corners instead of DoG keypoints. The proposed point matching technique finds more repeated features than the existing NNDR matching technique with the expense of little more computational time. However, this additional expense can be considered negligible because of high improvement in the identification accuracy (comparing accuracy of Approaches 2 and 4 in Table II). The SIFT descriptor was also experimentally proved to be more distinctive than the curvature descriptor (comparing matching time of Approaches 3 and 5 in Table II). Therefore, the two feature-point reduction solutions combined with the SIFT descriptors and the proposed featurepoint matching technique not only improve the computational efficiency and decrease the storage requirement, but also improve the TII accuracy.

\section{REFERENCES}

[1] M. Awrangjeb and M. Murshed, "Robust signature-based geometric invariant copyright protection," in Proc. Int. Conf. on Image Process. Atlanta, USA, 2006, pp. 1961-1964.

[2] D. G. Lowe, "Distinctive image features from scale-invariant keypoints," Int. Journal of Comp. Vision, vol. 60, no. 2, pp. 91-110, 2004.

[3] G. Lu, Multimedia Database Management Systems. Norwood: Artech House Inc., 1999.

[4] H. Bay, T. Tuytelaars, and L. V. Gool, "Surf: speeded up robust features," in Proc. European Conf. on Comp. Vision, vol. LNCS 3951, no. 1, Austria, 2006, pp. 404-417.

[5] C. Harris and M. Stephens, "A combined corner and edge detector," in Proc. Alv. Vis. Conf., 1988, pp. 147-151.

[6] T. Lindeberg, "Feature detection with automatic scale selection," Int. Journal of Comp. Vision, vol. 30, no. 2, pp. 77-116, 1998.

[7] K. Mikolajczyk and C. Schmid, "Scale \& affine invariant interest point detectors," Int. J of Comp. Vision, vol. 60, no. 1, pp. 63-86, Oct. 2004.

[8] M. Awrangjeb, G. Lu, and M. Murshed, "An affine resilient curvature scale-space corner detector," in Proc. Int. Conf. on Acoustics, Speech, and Signal Process., vol. 1, Hawaii, USA, Apr. 2007, pp. 1233-1236.

[9] F. Mokhtarian and R. Suomela, "Robust image corner detection through curvature scale space," IEEE Trans. on Patt. Anal. and Machine Intel., vol. 20, no. 12, pp. 1376-1381, 1998.

[10] K. Mikolajczyk and C. Schmid, "A performance evaluation of local descriptors," IEEE Trans. on Patt. Anal. and Machine Intel., vol. 27, no. 10, pp. 1615-1630, Oct. 2005.

[11] Y. Ke and R. Sukthantar, "Pca-sift: a more distinctive representation for local image descriptors," in Proc. Int. Conf. on Comp. Vision and Pattern Recognition, vol. 2, USA, Jun.-Jul. 2004, pp. 506-513.

[12] J. Canny, "A computational approach to edge detection," IEEE Trans. on Patt. Anal. and Machine Intel., vol. 8, no. 6, pp. 679-698, 1986.

[13] M. Brown and D. Lowe, "Invariant features from interest point groups,' in Proc. British Machine Vision Conf., UK, Sep. 2002, pp. 656-665.

[14] F. Klein, Elementary mathematics from an advanced standpoint: geometry. New York: Macmillan Company, 1939.

[15] M. Fischler and R. Bolles, "Random sample consensus: a paradigm for model fitting with applications to image analysis and automated cartography," Comm. of the ACM, vol. 24, no. 6, pp. 381-395, 1981.

[16] F. A. P. Petitcolas, "Watermarking schemes evaluation," IEEE Signal Processing, vol. 17, no. 5, pp. 58-64, Sep. 2000.

[17] USC-SIPI. (2007) The usc-sipi image database. [Online]. Available: http://sipi.usc.edu/database/

[18] D. Nister. (2007) Recognition database. [Online]. Available: http://www.vis.uky.edu/ dnister/ 